\title{
Marker imputation with low-density marker panels in Dutch Holstein cattle
}

\author{
Z. Zhang* and T. Druet ${ }^{*}{ }^{1}$ \\ *Unit of Animal Genomics, Faculty of Veterinary Medicine and Centre for Biomedical Integrative Genoproteomics, University of Liège, \\ B-4000 Liège, Belgium \\ †Fonds de la Recherche Scientifique-FNRS, B-1000 Brussels, Belgium
}

\section{ABSTRACT}

The availability of high-density bovine genotyping arrays made implementation of genomic selection possible in dairy cattle. Development of low-density single nucleotide polymorphism (SNP) panels will allow the extension of genomic selection to a larger portion of the population. Prediction of ungenotyped markers, called imputation, is a strategy that allows using the same low-density chips for all traits (and for different breeds). In the present study, we evaluated the accuracy of imputation with low-density genotyping arrays in the Dutch Holstein population. Five different sizes of genotyping arrays were tested, from 384 to 6,000 SNP. According to marker density, the overall allelic imputation error rate obtained with the program DAGPHASE, which relies on linkage disequilibrium and linkage, ranged from 11.7 to $2.0 \%$, and that obtained with the program CHROMIBD, which relies on linkage and the set of all genotyped ancestors, ranged from 10.7 to $3.3 \%$. However, imputation efficiency was influenced by the relationship between low-density and highdensity genotyped animals. Animals with both parents genotyped had particularly low imputation error rates: $<1 \%$ with 1,500 SNP or more. In summary, missing marker alleles can be predicted with 3 to $4 \%$ errors with approximately $1 \mathrm{SNP} / \mathrm{Mb}$ (approximately 3,000 markers). The CHROMIBD program proved more efficient than DAGPHASE only at lower marker densities or when several genotyped ancestors were available. Future studies are required to measure the effect of these imputation error rates on accuracy of genomic selection with low-density SNP panels.

Key words: imputation, dairy cattle, genotyping

\section{INTRODUCTION}

Genomic selection (Meuwissen et al., 2001) is now widely used in dairy cattle (de Roos et al., 2007; VanRaden, 2008; VanRaden et al., 2009). It relies on genotyping a livestock population for thousands of SNP

Received June 3, 2010.

Accepted August 13, 2010.

${ }^{1}$ Corresponding author: tom.druet@ulg.ac.be across the entire genome and using these SNP to estimate breeding values of any genotyped animal.

Recently, Habier et al. (2009) proposed genotyping some animals on lower density marker panels to make genomic selection more cost effective. Unlike strategies based on selection of a subset of SNP associated with selected traits, they suggested the use of SNP evenly spaced across the genome. Indeed, the first strategy, as implemented in Weigel et al. (2009), requires a different set of SNP for each trait, whereas with the strategy of Habier et al. (2009), the same set of SNP can be used for all traits. This strategy is now considered by most dairy cattle breeding organizations that implemented genomic selection. Such a low-density chip with 3,000 SNP has already been developed in the United States, and Illumina (San Diego, CA) made it commercially available in 2010 .

However, the use of low-density marker panels requires methods for transferring information from individuals genotyped at a higher density. Prediction of missing markers, or imputation, is one technique that allows the use of individuals genotyped on different marker panels. Imputation is already widely used in human genetics (Marchini et al., 2007; Marchini and Howie, 2008). However, in studies applied to human populations, commonly used marker arrays are of much higher density (>300,000 SNP), and imputation relies mostly on linkage disequilibrium (LD), whereas in livestock species, availability of extended pedigrees allows the use of linkage information for imputation. Druet and Georges (2010) and T. Druet and F. Farnir (University of Liège, Liège, Belgium, unpublished data) proposed 2 methods adapted for marker imputation with lowdensity SNP panels in animal and plant breeding. The objective of the present study was to test these 2 methods with low-density SNP on a Dutch Holstein dairy cattle data set.

\section{MATERIALS AND METHODS}

\section{Data}

A set of 4,734 Holstein dairy cattle individuals genotyped for 45,836 SNP markers on the CRV chip, a 
custom-made $60 \mathrm{~K}$ Illumina panel described in Charlier et al. (2008), was used in this study. A more complete description of this data set can be found in Druet et al. (2010).

To test imputation efficiency, the animals were assigned to 2 groups: reference individuals were genotyped on all markers, whereas the target animals were genotyped on lower density SNP panels (we erased markers not present on the low-density panel).

Different sizes of reference populations were tested (500, 1,000, 1,500, and 2,000 reference individuals). Animals with a higher number of genotyped descendants were preferentially included in the reference population (516 individuals had at least one genotyped descendant). The remaining reference individuals were chosen at random. Remaining animals $(4,234,3,734,3,234$, and 2,734$)$ were considered as target individuals.

\section{Creation of Low-Density Marker Panels}

To mimic low-density genotyping, different lowdensity panels were defined (see below) and genotypes of target animals were erased for the unselected markers. Five different sizes of marker panels were tested: 384, 768, 1,536, 3,000, and 6,000 SNP. These sizes were chosen according to Illumina GoldenGate and iSelect BeadChip technologies, which allow custom genotyping with 384 to $1,536 \mathrm{SNP}$ and with 3,000 to 60,000 SNP, respectively. The markers were selected to obtain a compromise between uniform marker density and high minor allelic frequency (MAF) with the following method. The number of markers per chromosome was obtained by multiplying the desired marker density (total number of markers divided by the size, in $\mathrm{Mb}$, of the genome) by the size of the chromosome (in Mb). Each chromosome was divided into equal segments based on the desired number of markers. Then, the marker with the highest MAF was selected in the first segment. For subsequent segments, the marker with the highest score combining MAF and distance with the marker retained in the previous segment was selected:

$$
\operatorname{score}(i)=\operatorname{MAF}(i) \times[\operatorname{ssize}-|\operatorname{ssize}-\operatorname{dist}(i)|],
$$

where $i$ is the index of the tested marker, ssize is the size of each segment, and dist $(i)$ is the distance between the tested marker and the selected marker in the previous segment (Matukumalli et al., 2009).

\section{Marker Imputation Method}

Haplotype Reconstruction. Haplotypes of reference and target individuals were first partially recon- structed based on linkage and Mendelian segregation rules with LinkPHASE (Druet and Georges, 2010). Then, haplotypes from reference individuals were fully reconstructed by using Beagle (Browning and Browning, 2007) iteratively with scale and shift parameters equal to 2.0 and 0.1 and DAGPHASE (Druet and Georges, 2010) as described in Druet and Georges (2010). In total, 20 such iterations were performed to estimate the directed acyclic graph (DAG) describing reference haplotypes. Linkage information was ignored for parents with 5 offspring or fewer, because Druet and Georges (2010) observed that this procedure reduced haplotyping errors.

Missing Marker Imputation. Finally, a file containing completely reconstructed haplotypes for reference individuals and partially reconstructed haplotypes (obtained from LinkPHASE) for target individuals was used for marker imputation. Two imputation programs were used: DAGPHASE (Druet and Georges, 2010) and CHROMIBD (T. Druet and F. Farnir, University of Liège, Liège, Belgium; unpublished data).

With DAGPHASE, linkage information is used when parents are genotyped: partial haplotypes of offspring are used to determine which haplotype was received from the parent, and marker alleles of the transmitted haplotype are then used to fill in the missing markers in the progeny. For other haplotypes, LD information is used by inferring the path of these haplotypes in the DAG, which summarizes all reference haplotypes. Probabilities of different paths are computed based on partial haplotypes (genotyped markers) with a hidden Markov model described in Druet and Georges (2010). Then, the marker alleles labeling the path in the DAG are used to impute missing marker alleles. The LD modeled in the DAG results from both recent and old ancestors. When the haplotype of a recent ancestor is present in the DAG, the partial haplotype will go through the same path as the haplotype of the ancestor over a long portion of the graph. Therefore, identification of the path will be precise and possible even with few genotyped markers. Portions of haplotypes transmitted from more distant ancestors are shorter and therefore require higher marker densities to be inferred precisely. With DAGPHASE, 2 outputs can be obtained: prediction of all the missing markers based on the most likely "hidden" chain (as in Druet and Georges, 2010) or prediction of posterior genotype probabilities based on the Baum-Welch algorithm as described in Druet et al. (2010).

The CHROMIBD program uses linkage and a hidden Markov model to estimate identity-by-descent probabilities (IBD) between a chromosome and parental chromosomes from genotyped ancestors. It relies only 
on the set of genotyped ancestors and LD is not used. Haplotypes of these ancestors and IBD probabilities are then used to predict posterior genotyped probabilities of missing markers (T. Druet and F. Farnir, University of Liège, Liège, Belgium; unpublished data).

\section{Evaluation of Imputation Efficiency}

When marker alleles are predicted, imputation efficiency was estimated by comparing imputed marker alleles and real marker alleles (this procedure slightly underestimates precision as real genotypes have some errors). The allelic imputation error rate per animal was then estimated as:

$$
\frac{1}{2 \times N} \sum_{j=1}^{N}\left|O\left(n_{i j}^{1}\right)-I\left(n_{i j}^{1}\right)\right|
$$

where $N$ is the number of markers, $O\left(n_{i j}^{1}\right)$ and $I\left(n_{i j}^{1}\right)$ are the observed (real genotypes) and imputed number of allele " 1 " for individual $i$ at marker $j$, and $2 \times N$ is the total number of imputed alleles.

When posterior genotype probabilities were estimated, the expected number of " 1 " alleles was first computed as

$$
E\left(n_{i j}^{1}\right)=2 \times P\left(G_{i j}=11\right)+1 \times P\left(G_{i j}=12\right)+0 \times P\left(G_{i j}=22\right),
$$

where $E\left(n_{i j}^{1}\right)$ is the expected number of allele "1" for individual $i$ at marker $j$, and $P\left(G_{i j}=k l\right)$ is the probability that individual $i$ carries marker alleles $k$ and $l$ at marker $j$. The allelic imputation error rate per animal was finally estimated as

$$
\frac{1}{2 \times N} \sum_{j=1}^{N}\left|O\left(n_{i j}^{1}\right)-E\left(n_{i j}^{1}\right)\right| .
$$

Imputation efficiency was measured on the 2,734 individuals that were never reference individuals to compare results obtained with the same set of individuals across all designs.

\section{RESULTS AND DISCUSSION}

In this work, 3 factors affecting imputation efficiency were studied: density of markers in the small chip, relationship between target and reference individuals, and number of reference individuals. The effect of each factor is dependent on the imputation method used (CHROMIBD or DAGPHASE).
Results are presented as allelic imputation error rates (proportion of incorrectly imputed marker alleles). Genotype imputation error rates (proportion of incorrectly imputed genotypes) are approximately equal to twice the allelic imputation error rates. With 384 markers, the ratio between error rates was approximately 1.90. This ratio consistently increased with number of markers and was approximately 1.98 with 6,000 markers (ratios were computed in designs with 1,000 and 2,000 reference individuals).

\section{Influence of Marker Density}

The main question when designing low-density marker panels is what imputation efficiency can be achieved for a given number of SNP on the small chip. Figure 1 presents mean imputation error rates obtained for small chips with 384 to 6,000 SNP (with 1,000 reference individuals). With both DAGPHASE and CHROMIBD, the curve describing imputation efficiency as a function of marker density presented a hyperbolic pattern with a steep decrease at low densities and a moderate decrease with a higher number of markers. Such a relationship was described previously by Druet et al. (2010). With DAGPHASE, allelic imputation error rates ranged from $11.7 \%$ with 384 SNP to $2.0 \%$ with 6,000 SNP. With CHROMIBD, relying only on linkage, the differences were smaller: from 10.7 to $3.3 \%$. For comparison, imputing genotypes based on the most likely genotypes or randomly according to genotype frequencies resulted in allelic imputation error rates of 20.0 and $27.0 \%$, respectively. With DAGPHASE (CHROMIBD), adding approximately 1,500 SNP decreased errors rates by $6.9 \%(5.4 \%)$ when the initial number of markers was equal to 384 and by only $1.7 \%(1.3 \%)$ when having 3,000 markers instead of 1,536. Similarly, using 3,000 markers instead of 384 markers dramatically decreased error rates by $8.6 \%$ (6.7\%), whereas adding 3,000 more markers reduced errors only by $1.1 \%(0.7 \%)$. To obtain overall mean allelic imputation error rates around $5 \%$, chips with 1,536 SNP or more should be used. Such error rates might still be too high to obtain an accurate estimation of breeding values with genomic selection. Panels with 3,000 SNP or more are necessary to get around a $3 \%$ error rate or less (with DAGPHASE). The relationship between imputation error rates and precision of genomic selection must be studied before defining optimal SNP panels. Technical and economical considerations must be taken into account to decide whether larger chips should be used.

The results presented for different SNP panels are overall results, averaged over all animals. However, im- 


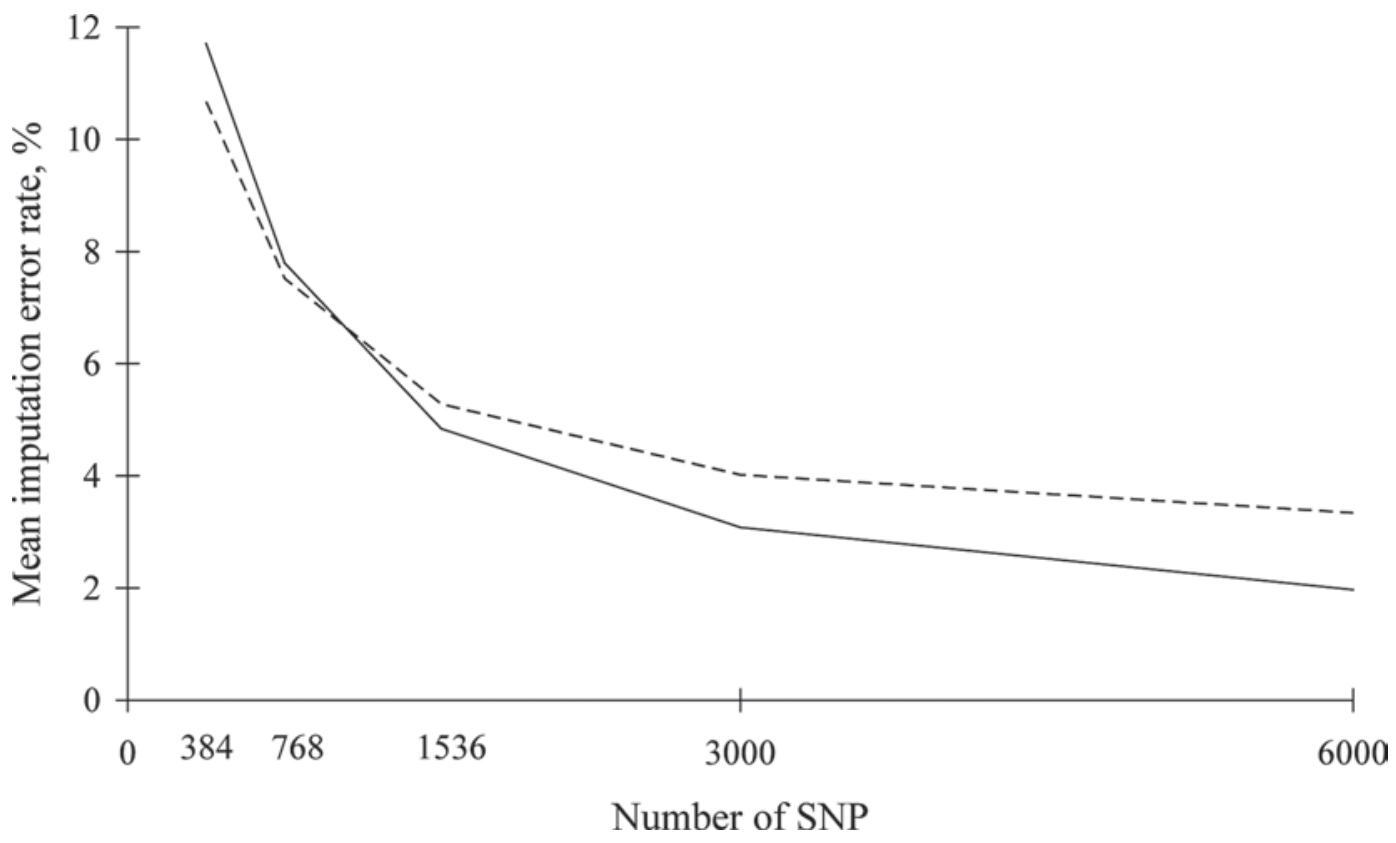

Figure 1. Mean allelic imputation error rate (\%) obtained for 1,000 reference individuals imputed with DAGPHASE (Druet and Georges, 2010; solid line) and CHROMIBD (T. Druet and F. Farnir, University of Liège, Liège, Belgium; dashed line) programs for different sizes of low-density panels.

putation accuracy is highly dependent on the relationship between target and reference individuals.

\section{Relationship Between Target and Reference Individuals}

Druet et al. (2010) and T. Druet and F. Farnir (University of Liège, Liège, Belgium; unpublished data) already showed that DAGPHASE and CHROMIBD result in higher imputation accuracy when the relationship between target and reference individuals is higher. This relationship was measured as a relationship score equal to the expected proportion of the genome inherited from reference individuals (scores of $0.5,0.75,0.875,0.9375$, and 0.96875 correspond to all male parents genotyped for $1,2,3,4$ and 5 generations, respectively). Such behavior is expected because CHROMIBD directly models IBD probabilities between the target and the reference individuals, whereas DAGPHASE uses haplotypes of reference individuals to estimate the optimal DAG. If more ancestors of an individual were used to construct the DAG, there is more chance that the haplotype of that individual is present in the DAG and over longer stretches (see Methods).

Table 1 contains the mean imputation error rates of DAGPHASE and CHROMIBD for all tested marker panels and different categories of relationships between target and reference individuals. The computed relationship scores ranged from 0.0 to 1.0 with the median equal to 0.9375 . In total, $94.8,84.6$, and $70.0 \%$ of the target individuals had a relationship score higher or equal to $0.75,0.85$, and 0.90 , respectively. When almost no ancestors were genotyped (relationship score $<0.50$ ), error rates ranged according to the marker density from 4.8 to $20.2 \%$ with DAGPHASE and from 22.3 to $26.8 \%$ with CHROMIBD. For higher relationship scores, imputation error rates decreased, particularly with CHROMIBD. For target individuals with a relationship score $>0.75$, DAGPHASE resulted in imputation error rates below 3 and $4 \%$ with 6,000 or 3,000 SNP, respectively. With CHROMIBD, such error rates were obtained only for target individuals with relationship scores $>0.90$.

For animals with both parents in the reference group (relationship score $=1$ ), the mean imputation rate was clearly lower than for other animals, ranging from 3.20 to $0.30 \%$ with DAGPHASE and from 4.20 to $0.40 \%$ with CHROMIBD. For these animals, haplotypes received from parents are inferred accurately by using linkage (for a single generation). For other animals, the maternal haplotype (and sometimes the paternal haplotype, too) is more difficult to model. The imputation error rate per animal is the mean from imputation error rates from paternal and maternal haplotypes. Because sires are most often genotyped, we can assume that imputation efficiency for paternal haplotypes is approximately equal to the imputation efficiency observed for animals with both parents in the reference group (because all 
Table 1. Allelic imputation error rates (\%) obtained with DAGPHASE and CHROMIBD programs ${ }^{1}$ for animals grouped by a score representing the expected proportion of the genome inherited from a reference individual (results are presented for different sizes of SNP panels from 384 to $6,000)$

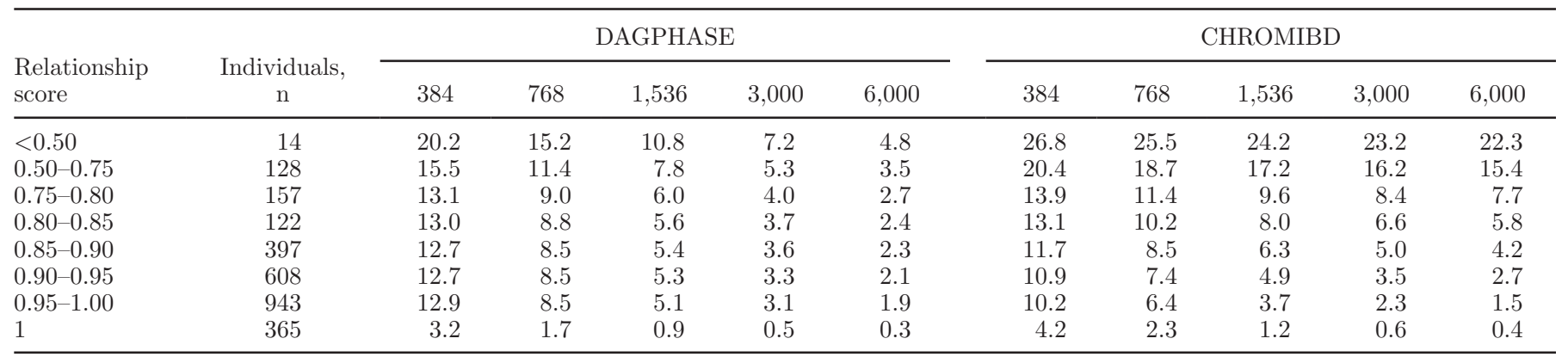

${ }^{1}$ DAGPHASE (Druet and Georges, 2010); CHROMIBD (T. Druet and F. Farnir, University of Liège, Liège, Belgium).

these haplotypes are described through linkage). Therefore, the imputation error rate for maternal haplotypes is probably higher (approximately equal to twice the error rate per animal minus the error rate observed when the relationship score equals 1 ).

\section{Influence of the Number of Individuals Genotyped in the Reference Panel}

Figure 2 describes the relationship between number of individuals genotyped on all markers and imputation efficiency with DAGPHASE or CHROMIBD for 5 different marker densities. With DAGPHASE, imputation efficiency constantly increased when the number of reference individuals increased: from 12.2 to $11.3 \%$, 8.8 to $6.9 \%, 5.9$ to $3.9 \%, 4.1$ to $2.3 \%$, and 2.7 to $1.4 \%$ with $384,768,1,536,3,000$, and 6,000 markers on the small chip, respectively. With CHROMIBD, almost no differences were observed when increasing size of the reference population. In comparison with the influence of the number of SNP or of the relationship between target and reference individuals, the effect of the number of individuals genotyped in the reference panel was small.

Druet et al. (2010) studied the relationship between number of reference individuals and imputation efficiency at higher marker densities. The benefit was more pronounced when very few reference individuals $(<500)$ were available. Above 1,000 genotyped reference individuals, only small gains in efficiency were observed (by improving estimation of LD between markers). In many dairy cattle populations, large numbers of individuals are already genotyped on high-density marker panels. Therefore, the size of the reference population should already be large enough and not be a major factor influencing imputation accuracy using small chips for a portion of the population. The main benefit of increasing number of reference individuals will be obtained through increasing relationship between target and reference individuals (see above).

\section{Comparison of Imputation Methods}

The results presented in earlier sections highlighted differences between DAGPHASE and CHROMIBD. First, CHROMIBD is more efficient than DAGPHASE with few SNP per panel, whereas DAGPHASE is better than CHROMIBD when panels have 1,536 SNP or more (Figure 1). Second, the improvement of imputation accuracy when the relationship between target and reference animals increases is more pronounced with CHROMIBD. With DAGPHASE, the imputation efficiency improves only slightly when the relationship score increases from 0.75 to 0.95 , whereas with CHROMIBD, the benefit of having additional genotyped ancestors still results in large differences in imputation efficiency. For low relationship scores, CHROMIBD is clearly less accurate than DAGPHASE; CHROMIBD results are better for high scores. However, the relationship score above which CHROMIBD achieves lower imputation error rates depends on the number of SNP on the small panel: at low density (384 SNP), CHROMIBD is better for animals with a relationship score $>0.85$, whereas at higher densities (6,000 SNP), using CHROMIBD is more efficient for animals with a relationship score $>0.95$. For animals with both parents genotyped, imputation performed with DAGPHASE had the lowest error rates. Indeed, in that situation, both methods use the same information (linkage) but DAGPHASE models both haplotypes jointly, whereas CHROMIBD models them independently. Finally, CHROMIBD was barely affected by size of the reference population, whereas having more reference individuals improved imputation with DAGPHASE.

All these observations can be explained by differences in the methods. DAGPHASE uses LD due to recent 


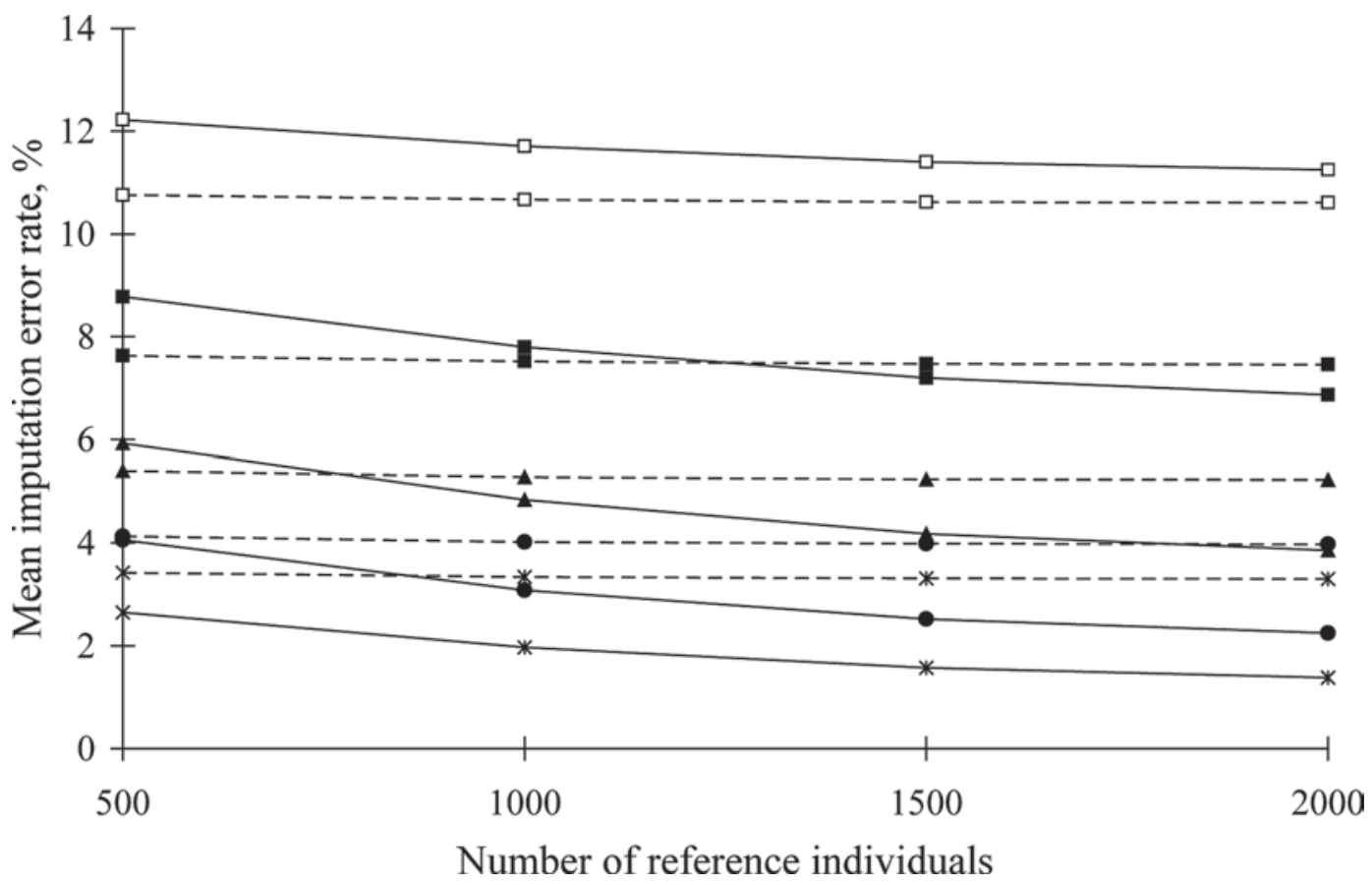

Figure 2. Mean allelic imputation error rate (\%) for low-density panel with $384(\square), 768(\boldsymbol{\square}), 1,536(\boldsymbol{\Lambda}), 3,000(\bullet)$, and 6,000 $(*)$ SNP obtained with DAGPHASE (Druet and Georges, 2010; solid line) and CHROMIBD (T. Druet and F. Farnir, University of Liège, Liège, Belgium; dashed line) programs for different sizes of reference population.

and old ancestors. Linkage disequilibrium is stronger at higher marker densities, and increasing size of reference population improves estimation of LD in the DAG. At low marker densities, LD is relatively weak and, as a result, imputation efficiency decreases. CHROMIBD models the IBD process along a chromosome between a target chromosome and a set of parental chromosomes. It relies, therefore, on recent ancestors that transmitted relatively long chromosome fragments and can work at lower marker densities. However, the efficiency is strongly influenced by the number of ancestors that are genotyped.

As mentioned in the Methods section, posterior genotype probabilities can also be obtained with DAGPHASE. Use of this output resulted in slightly higher imputation error rates (results not shown). However, use of posterior genotype probabilities (as with CHROMIBD, too) gives additional information beyond simply predicting likely genotypes. Indeed, the reliability of the imputation is known when using posterior probabilities and the user knows which marker alleles can be imputed with limited risk. It is, for instance, possible to call only marker alleles with posterior genotype probability above a certain threshold. In addition, these probabilities can be used to estimate the reliabilities of genomic predictions obtained with imputed markers. Finally, Weigel et al. (2010) mentioned that it might be advantageous to use genotype probabilities in genomic selection rather than calling genotypes.

In agreement with Druet and Georges (2010), results obtained with DAGPHASE show that it is beneficial to use both linkage and LD information for imputation rather than relying solely on LD (e.g., with Beagle). In Druet and Georges (2010), the algorithm of Beagle was found to be more efficient for marker imputation than the algorithm of fastPHASE (Scheet and Stephens, 2006). We also tested HiddenPHASE relying on the fastPHASE algorithm for description of LD (Druet and Georges, 2010), but imputation error rates and running times were higher (data not shown). Weigel et al. (2010) used fastPHASE (Scheet and Stephens, 2006) and IMPUTE (Marchini et al., 2007) to study the feasibility of imputation in Jersey cattle for different sizes of SNP panels for 3 chromosomes. Their imputation method relied purely on LD. When comparing results from Weigel et al. (2010) and those of the present study, different factors must be considered: the relationship between target and reference individuals (the authors mention that most animals had a relationship score $>0.75$ without further detail), the reference panel (2,542 Jersey animals), and LD within breed. Weigel et al. (2010) report that previous studies indicated that LD might be higher in Jersey than in Holstein (e.g., Villa-Angulo et al., 2009). With approximately 430, 870, 2,170, 4,340, 
and 8,680 SNP, Weigel et al. (2010) obtained, with their best method, genotype imputation error rates between 25.7 and $31.1 \%, 19.9$ and $27.3 \%, 9.5$ and $11.4 \%, 5.8$ and $8.3 \%$, and 3.6 and $5.8 \%$, respectively. In our studies, genotyping error rates (with 2,000 reference individuals and the best method) were equal to $20.2,13.0,7.4,4.3$, and $2.7 \%$ with $384,768,1,536,3,000$, and 6,000 SNP, respectively. These results indicate that, with fewer markers and reference individuals and probably lower LD, our method achieved higher imputation accuracy, although it is difficult to compare results from different data sets. Higher accuracy is explained by the fact that the method accounts for linkage information. The study of Weigel et al. (2010) was indeed designed to obtain lower bounds of imputation accuracy, because it relied on LD alone and ignored linkage information (Weigel et al., 2010). Use of the Beagle algorithm (or CHROMIBD) might also achieve better imputation accuracy than fastPHASE. In addition, computational costs of methods used in our study were lower (see below). With fastPHASE and IMPUTE, Weigel et al. (2010) mentioned computational problems and the need to break chromosomes in several pieces.

\section{Computational Requirements}

Computation times and memory requirements were measured on a computer cluster with Intel Xeon "Harpertown" L5420 processor at $2.50 \mathrm{GHz}$ (IBM Corp., Armonk, NY). Each chromosome was processed on its own CPU core. Computational requirements are presented for a design with 3,000 SNP on the low-density panel and 1,000 reference individuals. Estimation of parameters of the DAG and haplotype reconstruction of reference individuals required from 5 to $35 \mathrm{~min}$ per chromosome (Beagle was run with 2 GB memory, and DAGPHASE used less memory). For imputation with DAGPHASE, computation times per chromosome ranged from 2 min to $10 \mathrm{~min}$ and memory requirements per chromosome ranged from 275 to $620 \mathrm{MB}$ of memory. With CHROMIBD, computational requirements were higher: from 4 min to $15 \mathrm{~min}$ and from $420 \mathrm{MB}$ to 1.16 GB of memory.

When new genotypes are available, repeating the imputation should improve the accuracy of prediction of missing markers. However, repeating imputation for all animals has huge computational costs. To reduce these costs, the DAG should not be estimated at every imputation (unless the DAG has been initially estimated on a small sample) and a reference training set might be used for this purpose (not the whole genotyped population). In addition, haplotypes of reference individuals should be re-estimated only when new available information (such as new progeny or parents genotypes) is expected to significantly improve this process. Finally, when imputing a set of newly genotyped animals, only haplotypes of these animals and their genotyped parents are needed. Running the imputation on such a subset would be much faster and require less memory. Imputation should be redone for individuals only if accuracy would significantly increase for them (e.g., if a parent of the target individual has been newly genotyped or if haplotype reconstruction of a parent has been improved thanks to new genotyped offspring).

\section{CONCLUSIONS}

With both tested imputation methods, missing marker alleles can be predicted with 3 to $4 \%$ errors with approximately $1 \mathrm{SNP} / \mathrm{Mb}$ (approximately 3,000 markers). These figures can further be reduced by adding more markers. With DAGPHASE, these error rates can be achieved for animals with at least their sire and maternal grandsire genotyped. With CHROMIBD, 4 generations (or more) of male ancestors are required to obtain error rates $<4 \%$ (with 3,000 SNP). Such situations are fortunately frequent in the Dutch Holstein population. When both parents are genotyped, imputation error rates were $<1 \%$ with $1,500 \mathrm{SNP}$ or more. CHROMIBD is more efficient than DAGPHASE only at lower marker densities or when several genotyped ancestors are available. Future studies are required to measure the effect of these imputation error rates on accuracy of genomic selection with low-density SNP panels and to determine which animals should be genotyped on low-density chips to optimize breeding programs. In addition to genomic selection, use of low-density genotypes might also be beneficial in $\mathrm{F}_{2}$ designs used for QTL mapping; $\mathrm{F}_{0}$ animals would then be genotyped at high density and small chips would be used for later generations. In that case, imputation would be efficient because both parents are genotyped. Such a procedure could speed up QTL mapping and keep costs relatively low.

\section{ACKNOWLEDGMENTS}

Zhiyan Zhang has a PhD fellowship from the China scholarship council. Tom Druet is research associate from the F.R.S.-FNRS (Brussels, Belgium). The authors thank CRV (Arnhem, the Netherlands) for access to their data. This work was funded by grants of the Service Public de Wallonie (Jambes, Belgium) and from the Communauté Française de Belgique (Brussels, Belgium) (Biomod ARC). We acknowledge University of Liège (Liège, Belgium) (SEGI and GIGA bioinformatics platform) for the use of NIC3 and GIGA-grid supercomputers. 


\section{REFERENCES}

Browning, S. R., and B. L. Browning. 2007. Rapid and accurate haplotype phasing and missing-data inference for whole-genome association studies by use of localized haplotype clustering. Am. J. Hum. Genet. 81:1084-1097.

Charlier, C., W. Coppieters, F. Rollin, D. Desmecht, J. S. Agerholm, N. Cambisano, E. Carta, S. Dardano, M. Dive, C. Fasquelle, J. C. Frennet, R. Hanset, X. Hubin, C. Jorgensen, L. Karim, M. Kent, K. Harvey, B. R. Pearce, P. Simon, N. Tama, H. Nie, S. Vandeputte, S. Lien, M. Longeri, M. Fredholm, R. J. Harvey, and M. Georges. 2008. Highly effective SNP-based association mapping and management of recessive defects in livestock. Nat. Genet. 40:449-454.

de Roos, A. P., C. Schrooten, E. Mullaart, M. P. Calus, and R. F. Veerkamp. 2007. Breeding value estimation for fat percentage using dense markers on Bos taurus autosome 14. J. Dairy Sci. 90:4821-4829

Druet, T., and M. Georges. 2010. A hidden Markov model combining linkage and linkage disequilibrium information for haplotype reconstruction and QTL fine mapping. Genetics 184:789-798.

Druet, T., C. Schrooten, and A. P. W. de Roos. 2010. Imputation of genotypes from different single nucleotide polymorphism panels in dairy cattle. J. Dairy Sci. 93:5443-5454. doi:10.3168/jds.20103255

Habier, D., R. L. Fernando, and J. C. Dekkers. 2009. Genomic selection using low-density marker panels. Genetics 182:343-353.

Marchini, J., and B. Howie. 2008. Comparing algorithms for genotype imputation. Am. J. Hum. Genet. 83:535-539, 539-540.

Marchini, J., B. Howie, S. Myers, G. McVean, and P. Donnelly. 2007. A new multipoint method for genome-wide association studies by imputation of genotypes. Nat. Genet. 39:906-913.
Matukumalli, L. K., C. T. Lawley, R. D. Schnabel, J. F. Taylor, M. F. Allan, M. P. Heaton, J. O'Connell, S. S. Moore, T. P. Smith T. S. Sonstegard, and C. P. Van Tassell. 2009. Development and characterization of a high density SNP genotyping assay for cattle. PLoS ONE 4:e5350.

Meuwissen, T. H., B. J. Hayes, and M. E. Goddard. 2001. Prediction of total genetic value using genome-wide dense marker maps. Genetics 157:1819-1829.

Scheet, P., and M. Stephens. 2006. A fast and flexible statistical model for large-scale population genotype data: Applications to inferring missing genotypes and haplotypic phase. Am. J. Hum. Genet. 78:629-644

VanRaden, P. M. 2008. Efficient methods to compute genomic predictions. J. Dairy Sci. 91:4414-4423.

VanRaden, P. M., C. P. Van Tassell, G. R. Wiggans, T. S. Sonstegard, R. D. Schnabel, J. F. Taylor, and F. S. Schenkel. 2009. Invited review: Reliability of genomic predictions for North American Holstein bulls. J. Dairy Sci. 92:16-24.

Villa-Angulo, R., L. K. Matukumalli, C. A. Gill, J. Choi, C. P. Van Tassell, and J. J. Grefenstette. 2009. High-resolution haplotype block structure in the cattle genome. BMC Genet. 10:19.

Weigel, K. A., G. de los Campos, O. Gonzalez-Recio, H. Naya, X. L. Wu, N. Long, G. J. Rosa, and D. Gianola. 2009. Predictive ability of direct genomic values for lifetime net merit of Holstein sires using selected subsets of single nucleotide polymorphism markers. J. Dairy Sci. 92:5248-5257.

Weigel, K. A., C. P. Van Tassell, J. R. O'Connell, P. M. VanRaden, and G. R. Wiggans. 2010. Prediction of unobserved single nucleotide polymorphism genotypes of Jersey cattle using reference panels and population-based imputation algorithms. J. Dairy Sci. 93:2229-2238. 